Supporting Information

\title{
Re-engineering electrochemical aptamer-based biosensors to tune their useful dynamic range via distal-site mutation and allosteric inhibition
}

\author{
Shaoguang Li, Chengcheng Li, Yuanyuan Wang, Hui Li*, Fan Xia
}

Engineering Research Center of Nano-Geomaterials of Ministry of Education, Faculty of Materials Science and Chemistry, China University of Geosciences, Wuhan 430074, China

lihui-chem@cug.edu.cn 


\section{Table of Content}

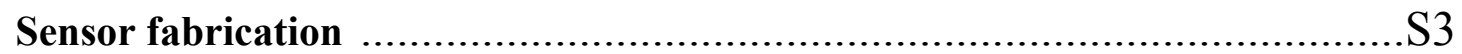

Kinetics and thermal stability measurements ....................................S4

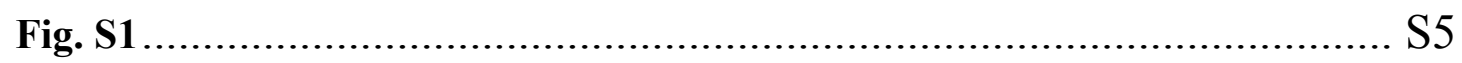

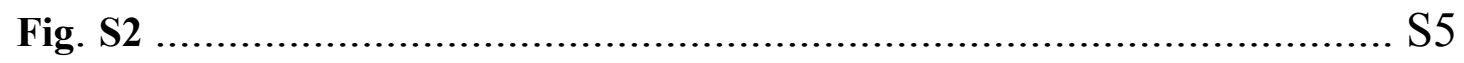

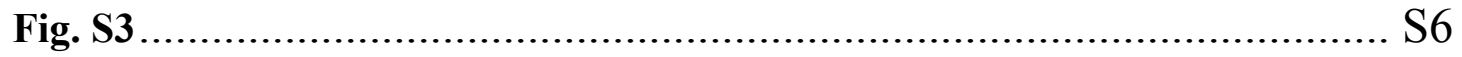

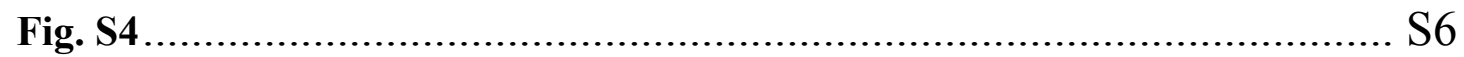

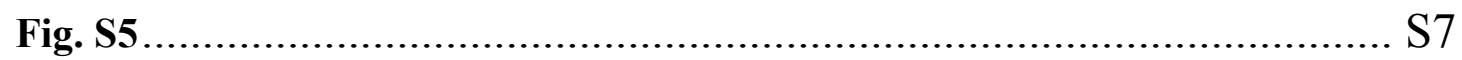


Sensor fabrication We followed the same procedure for sensor fabrication as we previously reported. Gold wire electrodes were made using the following procedure: the wire is composed of two parts: gold wire $(7-8 \mathrm{~mm}, 0.2 \mathrm{~mm}$ diameter $)$ and tungsten wire ( $8 \mathrm{~cm}, 0.2 \mathrm{~mm}$ diameter). These two components were first cold soldered using electrically conductive silver epoxy adhesive. The tungsten wire was then insulated using a heat shrinkable teflon tubing leaving the gold wire exposed for DNA modification and $1 \mathrm{~cm}$ tungsten wire at the other end for connection to the potentiostat. Following the insulation step, the gold portion of these was electrochemically roughened in order to increase the surface area. Briefly, the sensors were immersed in $0.5 \mathrm{M}$ sulfuric acid and rapidly pulsed between $\mathrm{E}_{\text {initial }}=0.0 \mathrm{~V}$ to $\mathrm{E}_{\text {high }}=2.0 \mathrm{~V}$ vs $\mathrm{Ag} / \mathrm{AgCl}$ for 400,000 times with each pulse being of $2 \mathrm{~ms}$ duration. Immediately prior to sensor fabrication we prepared a solution of thiol-and-methylene-blue-modified DNA in phosphate buffered saline buffer (PBS;(1×) (10.00 mM Na $2 \mathrm{HPO}_{4} ; 1.75 \mathrm{mM}$ $\left.\mathrm{KH}_{2} \mathrm{PO}_{4} 137.00 \mathrm{mM} \mathrm{NaCl} ; 2.65 \mathrm{mM} \mathrm{KCl} ; \mathrm{pH}=7 \sim 8\right)$ ) by incubating a solution of 100 $\mu \mathrm{M}$ DNA and $20 \mathrm{mM}$ tris-(2-carboxyethyl) phosphine hydrochloride (1:200) for $1 \mathrm{hr}$ at room temperature followed by dilution with PBS to $200 \mathrm{nM}$ as confirmed by UV-V is spectroscopy. We then immersed freshly cleaned electrodes in this solution for $1 \mathrm{hr}$ at room temperature. The resulting sensors were washed with deionized water and then incubated in $20 \mathrm{mM}$ 6-Mercapto-1-hexanol(MCH) solution in PBS overnight at $4^{\circ} \mathrm{C}$ before being rinsed with water prior to use. For the dynamic range measurement employing depletant mechanism, first we fabricated the sensors using a total concentration of $200 \mathrm{nM}$, in which the molar ratio of variant 1 and depletant (unlabeled 
parent aptamer) being 1: 10 and 1:25 respectively. For the measurements to broaden dynamic range, we employed a mixture of parent aptamer (200 nM), inhibitor 10 (30 $\mathrm{nM})$, and inhibitor $14(30 \mathrm{nM})$ to fabricate the sensor.

\section{Kinetics and thermal stability measurements}

All measurements in the kinetic and stability study was conducted after 30 times prescan to ensure the stability of E-AB sensors in PBS buffer. We then measured the electrochemical signals upon the addition of inhibitors. Once it reaches the equilibrium (max. $30 \mathrm{~min}$ ), we removed the unbound, free inhibitor in the solution, and replaced with a fresh buffer. The signal remaining is related to the stability of this inhibitoraptamer complex. Upon the removal of inhibitor in the solution, the sensor employing inhibitor 10 exhibited a recovery of current, indicating of a slowly de-hybridization process. The sensors employing inhibitor 12 and 14, in contrast, exhibited no current change, meaning that the complexes remain stable under such conditions. Thus, in the inhibition dynamic range study, we titrated the sensors in the presence of unbound, free inhibitors. 


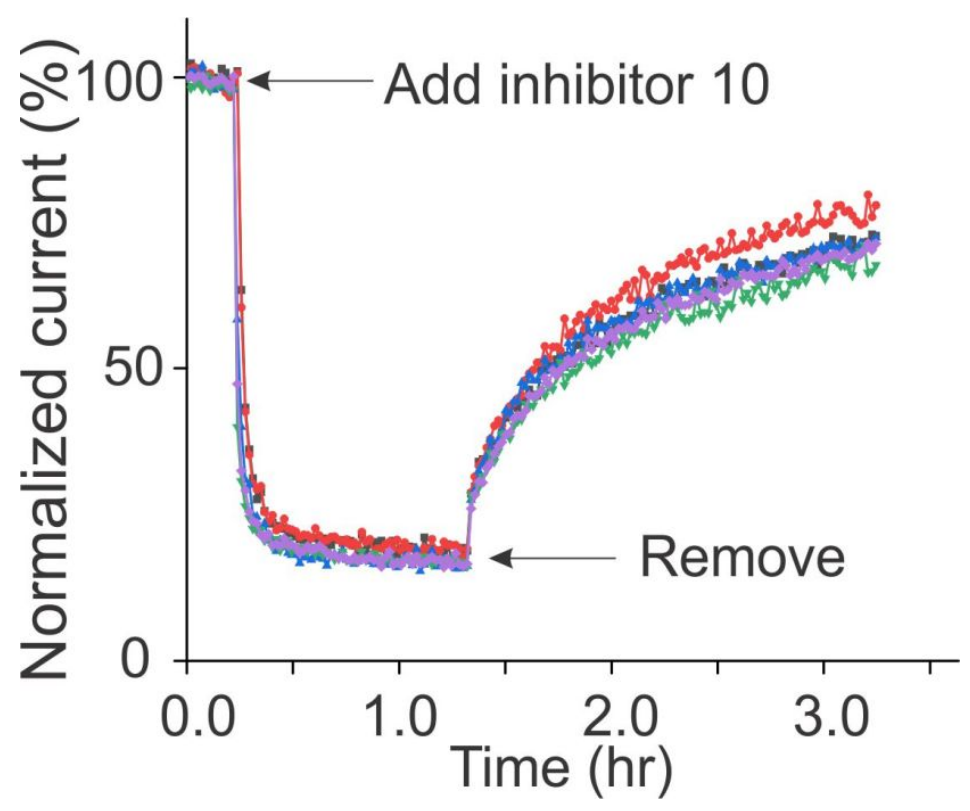

Fig. S1 The thermal stability of hybridized complex of parent and inhibitor is greatly dependent on the length of the inhibitor. For example, the shortest inhibitor (10mer) produces a hybridized duplex complex with parent aptamer, showing a slowly recovering current upon the removal of free inhibitor in solution (the "remove" as an indicative of removing free inhibitor).

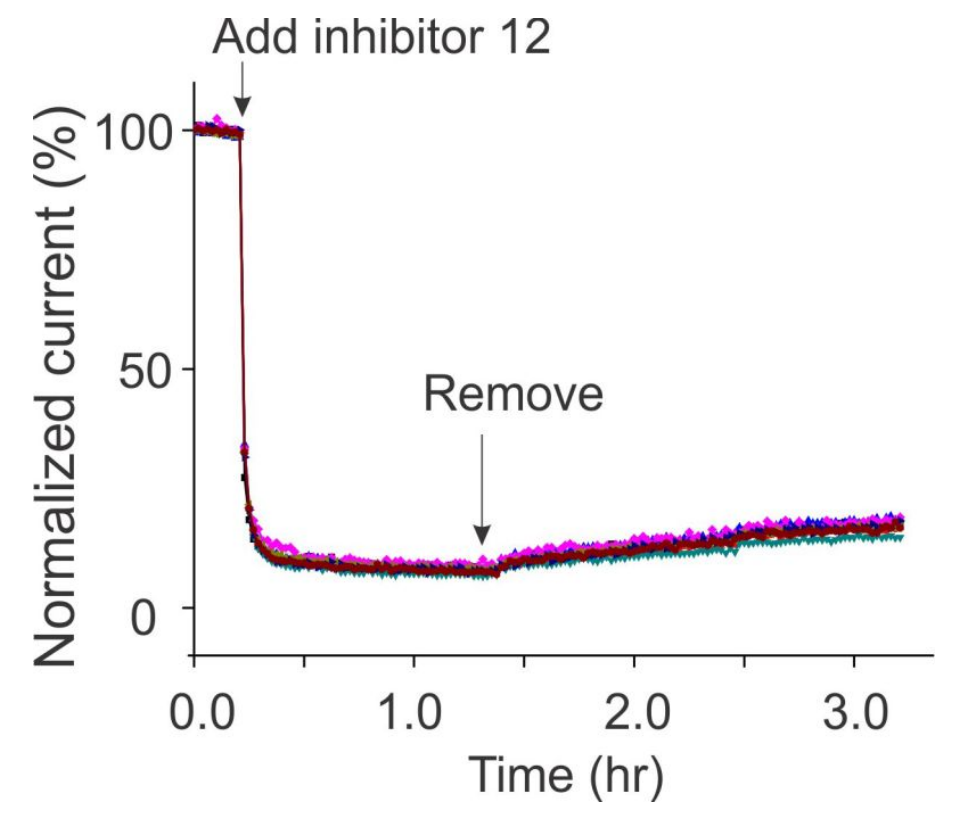

Fig. S2 In contrast, the complex of inhibitor 12 and aptamer exhibited a greatly improved thermal stability, with the signal remaining almost unchanged after the removal of free inhibitor in the solution. 


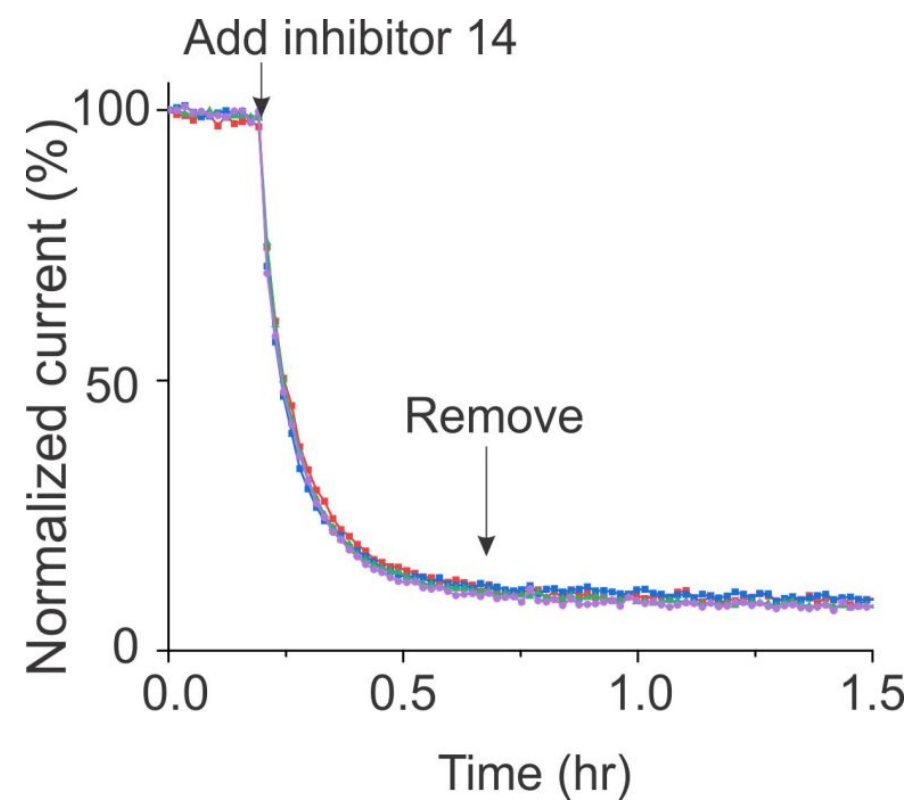

Fig. S3 Likewise, the complex of inhibitor 14 and aptamer exhibited a greatly improved thermal stability in comparison to 10 mer complex, with the signal remaining almost unchanged after the removal of free inhibitor in the solution.

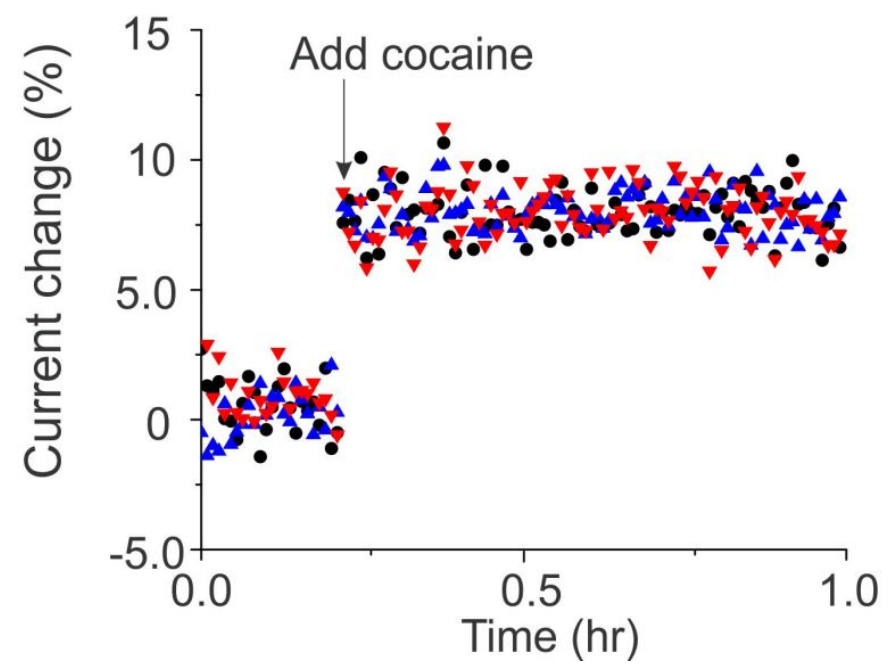

Fig. S4 The binding kinetics of inhibitor-aptamer complex towards its target molecule. Upon adding the target molecules, the sensors respond rapidly, reaching a maximum of signal change within duration of 30 seconds. 


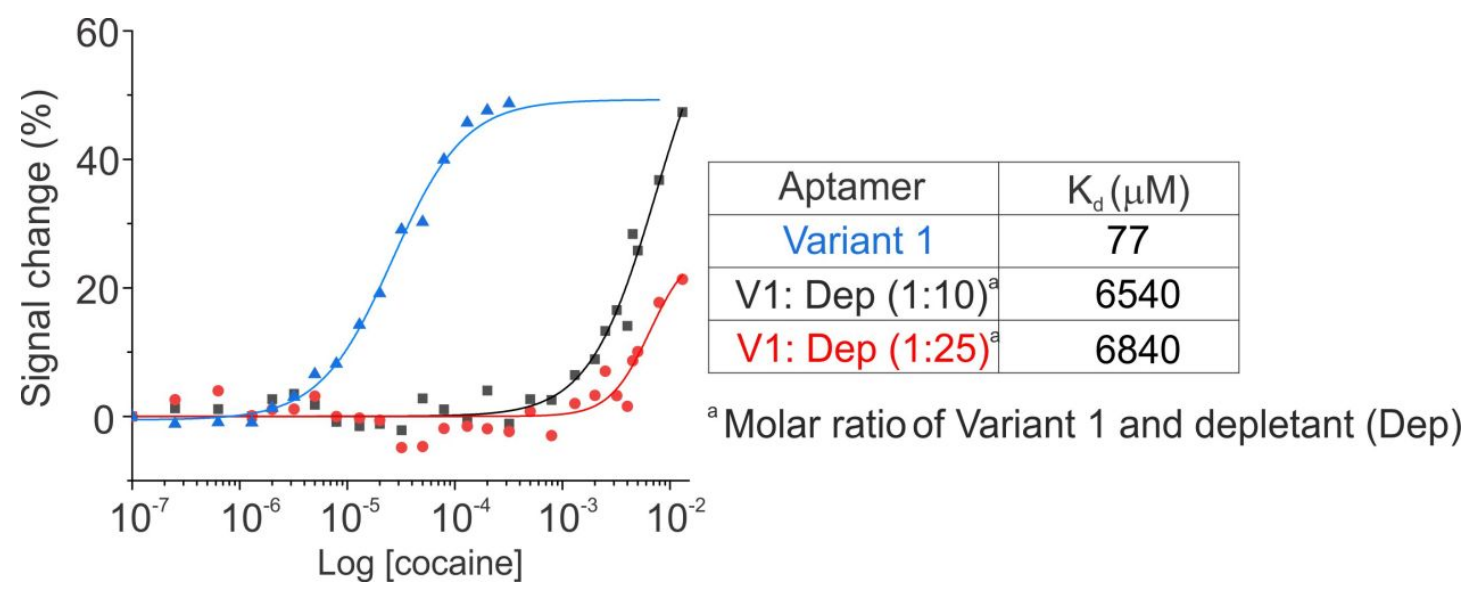

Fig. S5 We employed sequestration mechanism to narrow the dynamic range. Specifically, using variant 1 (V1 in short) as signaling aptamer, and depletant sequence (here unlabeled parent aptamer), we achieved a 3-fold dynamic range, versus a classic 81-fold for aptamer with a single binding site. In addition, the raw signal changes are related to the composition of aptamer on the surface, with more depletant producing smaller signal change. 